\title{
Noether Currents of Charged Spherical Black Holes
}

\author{
M. C. Ashworth* \\ Department of Physics, Tokyo Institute of Technology, Ohokayama, Meguro, Tokyo 152-8551, Japan \\ Sean A. Hayward, ${ }^{\dagger}$ \\ Center for Gravitational Physics and Geometry, 104 Davey Laboratory, The Pennsylvania State University, University Park, \\ PA 16802-6300, U.S.A.
}

(7th April 2000)

\begin{abstract}
We calculate the Noether currents and charges for Einstein-Maxwell theory using a version of the Wald approach. In spherical symmetry, the choice of time can be taken as the Kodama vector. For the static case, the resulting combined Einstein-Maxwell charge is just the mass of the black hole. Using either a classically defined entropy or the Iyer-Wald selection rules, the entropy is found to be just a quarter of the area of the trapping horizon. We propose identifying the combined Noether charge as an energy associated with the Kodama time. For the extremal black hole case, we discuss the problem of Wald's rescaling of the surface gravity to define the entropy.
\end{abstract}

It is widely excepted that black holes have entropy. However, without a full quantum theory of gravity, the statistical origin of this entropy is still unclear. On a classical and semi-classical level there have been many proposals discussing where this entropy comes from and ways of calculating it: spin structures, entanglements, edge states, etc. In particular interest for this paper is the Noether current calculation of Wald and Iyer [1]- [3]. The question becomes one of how do we test these proposals with our current level of understanding of gravity and quantum gravity? To date most of these calculations have been done in static or in some sense quasi-static cases [4. However, such cases may not reflect the general nature of the black hole. What is needed is further test cases, in particular dynamical test cases.

In this regards, spherically symmetric space-times provides us with a suitable dynamical testing ground. In the spherically symmetric case, while remaining dynamical, we can identify certain features defined in static cases. The time-like Killing vector that is identified as time in the static case can be replaced with the Kodama vector, $k=\star d r$ where $r=\sqrt{\mathcal{A} / 4 \pi}$ is the areal radius and $\star$ is the Hodge operator of the 2D normal space [5]. In addition, a local active gravitational energy can be defined by the Misner-Sharp energy [6],

\footnotetext{
*ashworth@th.phys.titech.ac.jp

${ }^{\dagger}$ hayward@gravity.phys.psu.edu
}

$$
E=\frac{r}{2}(1-d r \cdot d r)
$$

For dynamical space-times, a locally defined horizon and the global event horizon are generally not the same. Therefore, we must choose a suitable definition of the outer surface of the black hole. In the spherically symmetric setting, the outer surface of the black hole is proposed to be the trapping horizon defined by $\nabla r$ being null everywhere on the horizon [5]. In such a case, the energy on the trapping horizon $E$ is just half the areal radius, a natural generalization of the Schwarzschild radius.

Identifying these properties of the black-hole spacetime, it is possible to study the thermodynamics in a classical setting. The equations of motion can be shown to give an energy balance equation [5],

$$
\nabla E=\mathcal{A} \psi+w \nabla \mathcal{V}
$$

where $\psi$ is a localization of the Bondi energy flux, $w$ is an energy density and $\mathcal{V}=\frac{4}{3} \pi r^{3}$ is the areal volume. Looking more closely, we can identify the second term on the right-hand side as a work term. The first term on the right-hand side is an energy supply. This term, again using the equations of motion, can then be written as

$$
\mathcal{A} \psi=\frac{\kappa \nabla \mathcal{A}}{8 \pi}+r \nabla\left(\frac{E}{r}\right),
$$

where the dynamical surface gravity is defined as for stationary surface gravity by replacing the Killing vector with the Kodama vector [5], yielding $\kappa=\star d k / 2$. The last term vanishes when projected along a trapping horizon. If $\kappa / 2 \pi$ is the temperature on the trapping horizon, then the entropy is given by the area of the trapping horizon as $\mathcal{A} / 4$.

So, in a spherically symmetric system, we can identify the state variables of the model. The kinematical quantities are given by the areal radius and dynamical time $(r, k)$. The gravitational and matter quantities are $(E, \kappa)$ and $(w, \psi)$ respectively. Normally because there is no preferred time, such quantities are difficult to define.

In this testing ground, we would like to look at the Noether current calculations of Wald and Iyer in more detail. In general, gravitational theories are defined from diffeomorphism invariant actions, specified by a Lagrangian $n$-form $L[\phi]$, where $\phi$ denotes the dynamical fields including the space-time metric. For every such 
Lagrangian, there is an associated conserved current and charge, as follows, simplifying Wald's method by considering only perturbations $\delta$ which are Lie derivatives $\mathcal{L}_{\xi}$ along a vector $\xi$, which is the local generator of the diffeomorphisms. Then

$$
\delta L=* \Phi \circ \delta \phi+d \Theta
$$

defines the boundary $(n-1)$-form $\Theta[\phi, \xi]$, where $*$ is the space-time Hodge operator. The bulk term $\Phi$, which gives the equations of motion, has tensorial indices dual to $\phi$, with $\circ$ denoting contraction of all indices. This leads to a current $(n-1)$-form

$$
J=\Theta-\xi \cdot L
$$

Then the identity

$$
\mathcal{L}_{\xi} \Lambda=\xi \cdot d \Lambda+d(\xi \cdot \Lambda)
$$

implies

$$
d J=-* \Phi \circ \mathcal{L}_{\xi} \phi
$$

which vanishes when the equations of motion hold, $\Phi=0$. On shell $J$ is closed, and

$$
J=d Q
$$

defines a conserved charge $(n-2)$-form $Q$, up to various gauge freedom.

Originally for the Einstein action, Iyer and Wald 27 found that integrating the charge associated with the Killing time, after rescaling the surface gravity to one over the bifurcation surface, gave the known entropy of the static black hole. In the spherical setting, we can replace the Killing time with the Kodama vector as the diffeomorphism generator in the time direction. Integrating the charge from the Einstein action $L_{E}=* R / 16 \pi$ over a section of the trapping horizon instead of the bifurcation surface, we get the same form [7]

$$
\oint Q_{E}=\frac{\mathcal{A} \kappa}{8 \pi}
$$

In the static case when $k$ commutes with $d r$ the Kodama vector reduces to the Killing time [5], recovering the Wald-Iyer result; an entropy that is just the area of the horizon. However, we have now moved off the bifurcation surface to the locally defined trapping horizon.

In general, $\kappa$ is dynamical, the surface temperature of the black hole varying with time as the black hole area changes, so such rescaling of the charge seems somewhat artificial, as pointed out in [7]. So the question that then comes to mind is: what is this conserved current and

\footnotetext{
${ }^{1}$ Note that in Jacobson et al. [8] showed that any section of a Killing horizon is equivalent to the bifurcation surface.
}

charge? To get a broader look at this question, we should add matter fields to that action. So, let us consider the case that the matter fields are given by the Maxwell electromagnetic action,

$$
L_{M}[g, A]=-\frac{1}{16 \pi} * F: F,
$$

where the 1-form $A$ is the electromagnetic potential, $F=$ $2 d A$ and : is the trace of the dot product. Then we find

$$
\delta L_{M}=*(T: \delta g / 2+\Psi \cdot \delta A)+d \Theta
$$

where

$$
\begin{aligned}
& T=-(F \cdot F+(F: F) g / 4) / 4 \pi \\
& \Psi=* d * F / 4 \pi \\
& \Theta=-\delta(* A \cdot F) / 8 \pi .
\end{aligned}
$$

The resulting Noether current is

$$
J_{M}=d Q_{M}-\xi \cdot *(\Psi \cdot A) / 2,
$$

where the associated charge is

$$
Q_{M}=-\frac{1}{8 \pi} \xi \cdot *(A \cdot F) .
$$

Note that this method differs from that of Wald in that it has not been necessary to express $\Theta$ explicitly as a function of $(\phi, \delta \phi)$, thereby saving calculation. The calculation also uses the fact that $d$ and $\mathcal{L}_{\xi}$ commute. Note: this charge is dependent on the gauge choice of the EM fields, which is related to the Aharonov-Bohm effect.

We propose defining the energy of a field as twice the charge $Q$ integrated over an $(n-2)$-dimensional surface. For the Reissner-Nordström case, with the natural gauge choice $A=-e d t / r$, this gives the electromagnetic energy

$$
E_{M}=\oint 2 Q_{M}=\frac{e^{2}}{r}
$$

Combining this with the twice the Noether charge from the Einstein gravitational action (9),

$$
E_{E}=\oint 2 Q_{E}=\frac{\mathcal{A} \kappa}{4 \pi},
$$

gives a combined energy charge,

$$
E=E_{E}+E_{M}=m,
$$

that is just the mass of the black hole.

In [9], the question of the physical meaning of the total Noether current is brought up. The charge normally associated with transformations along a time direction is of course the energy. Although further cases need to be studied, in the Schwarzschild case and now the ReissnerNordström case, the total Noether charge is indeed the energy at the boundary. 
Another point about the Noether currents is that because the symplectic potential and the charge are only defined up to total derivatives, it is possible to change the charge by adding various surface terms. Iyer and Wald have proposed methods for choosing which terms should lead to the entropy [3], which does give the right entropy in the Reissner-Nordström case after rescaling the surface gravity. However, it seems easier to understand the freedom of the choice of the symplectic potential if the Noether charge is viewed as an energy. The changes in the current are analogous to Legendre transformations in thermodynamics which result in different energies such as the Gibb's free energy. So with Iyer and Wald's selection rule [3], the gravitational entropy seems to be singled out such that it occurs with only the surface gravity appearing in front of it.

In the above calculations, it is assumed that the spacetime is the normal black hole space-time resulting from $e^{2}<m^{2}$. In the case that $e^{2}>m^{2}$, there is no trapping horizon and no other inner boundary other than the singularity at $r=0$. In the extremal case $e^{2}=m^{2}$, the inner and outer trapping horizons coincide and become degenerate. Using standard null coordinates [10],

$$
d s^{2}=-\left(\frac{(r-m)^{2}}{r^{2}}\right) d x^{+} d x^{-}+r^{2} d \Omega^{2}
$$

where the areal radius is defined indirectly by

$$
\frac{1}{2}\left(x^{+}-x^{-}\right)=r+2 m \log (r-m)-\frac{2 m^{2}}{r-m}
$$

it is easy to see that $r=m$ is a still a well defined trapping horizon. However, the surface gravity is proportional to the difference between the inner and outer trapping horizon radius, $\kappa \propto\left(r_{+}-r_{-}\right)$, and is zero in the extremal case. In Wald's definition, the surface gravity must be rescaled to unity in order to define the entropy. In the extremal case, because the surface gravity is zero, it is not possible to do this rescaling, resulting in an illdefined entropy. Using the classical first law (3), it would still seem that the entropy is just the area of the trapping horizon over 4. However, using Nernst's theorem: if the temperature (the surface gravity) vanishes then the system settles in its ground state and the entropy vanishes. The system being in some sort ground state makes sense because the Einstein energy vanishes, $E_{E}=0$. The problem of the entropy of the extremal case has been discussed in various papers, for example [11]. However without a quantum-statistical model for the entropy, this problem cannot be resolved.

Acknowledgements. MCA is supported by NSF/JSPS Postdoctoral Fellowship for Foreign Researchers (No. P97198). SAH is supported by National Science Foundation award PHY-9800973.
[1] Robert M. Wald, Phys. Rev. D 48, 3427-3431 (1993).

[2] Vivek Iyer, Robert M. Wald, Phys.Rev. D 52, 4430-4439 (1995).

[3] Vivek Iyer and Robert M. Wald, Phys.Rev. D 50, 846864 (1994).

[4] A. Ashtekar, J. Baez, A. Corichi, and K. Krasnov, Phys. Rev. Lett. 80, 904 (1998); J. Gegenberg, G. Kunstatter, and D. Louis-Martinez, Phys. Rev. D51, 1781 (1995); S. Carlip, Class. Quantum Grav. 12, 2853 (1995); S. Carlip, Phys.Rev.Lett. 82, 2828-2831 (1999).

[5] Sean Hayward, Class.Quant.Grav. 15, 3147-3162 (1998).

[6] C. W. Misner and D. H. Sharp, Phys. Rev. B 571, 136 (1964).

[7] S. A. Hayward, S. Mukohyama, M. C. Ashworth, Phys.Lett. A256, 347-350 (1999).

[8] T. Jacobson, G. Kang, R. C. Myers, Phys. Rev. D49 6587-6598 (1994).

[9] M. C. Ashworth and S. A. Hayward, Phys.Rev. D60, 084004 (1999).

[10] S. W. Hawking and G. F. R. Ellis, The Large Scale Structure of Space-time (Cambridge University Press, 1973).

[11] S. W. Hawking, G. T. Horowitz, and S. F. Ross, Phys.Rev. D51 4302-4314 (1995). 